\title{
CHRNA3 genotype, nicotine dependence, lung function and disease in the general population
}

\author{
Diljit Kaur-Knudsen, Børge G. Nordestgaard and Stig E. Bojesen
}

ABSTRACT: The CHRNA3 rs1051730 polymorphism has been associated to chronic obstructive pulmonary disease (COPD), lung cancer and nicotine dependence in case-control studies with high smoking exposure; however, its influence on lung function and COPD severity in the general population is largely unknown.

We genotyped 57,657 adult individuals from the Copenhagen General Population Study, of whom 34,592 were ever-smokers. Information on spirometry, hospital admissions, smoking behaviour and use of nicotinic replacement therapy was recorded.

In homozygous (11\%), heterozygous (44\%) and noncarrier (45\%) ever-smokers, forced expiratory volume in $1 \mathrm{~s}$ (FEV1) was $94.1 \%$ predicted, $95.3 \%$ pred and $96.5 \%$ pred, forced vital capacity (FVC) was $97.1 \%$ pred, $97.5 \%$ pred and $98.3 \%$ pred, and FEV $1 / F V C$ was $0.770,0.773$ and $\mathbf{0 . 7 7 7}$, respectively (all $\mathrm{p}<\mathbf{0 . 0 0 1}$ for trend). Smoking interacted with genotype on $\mathrm{FEV}_{1} \%$ pred and FEV1/FVC (both $p<0.001$ ). When adjusted for cumulative tobacco consumption, these associations remained significant. In ever-smokers, odds ratios for COPD in homozygotes versus noncarriers were $1.3(95 \% \mathrm{Cl} 1.2-1.4)$ for Global Initiative for Chronic Obstructive Lung Disease (GOLD) stages I-IV, 1.4 (95\% CI 1.2-1.6) for GOLD II-IV and 1.7 (95\% CI 1.3-2.1) for GOLD III-IV. The corresponding value for lung cancer was 1.8 (95\% CI 1.2-2.6). Genotype was also associated with daily and cumulative tobacco consumption and with use of nicotinic replacement therapy in former smokers.

In ever-smokers, the CHRNA3 rs1051730 genotype associated with reduced lung function and increased COPD severity.

KEYWORDS: Chronic obstructive pulmonary disease, genetics, nicotinic acetylcholine receptor, smoking, spirometry

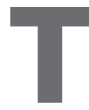
he CHRNA3 gene encoding the neuronal nicotinic acetylcholine receptor has been associated with lung function and chronic obstructive pulmonary disease (COPD) in a genome-wide association study (GWAS), with the strongest signal for the rs1051730 genotype [1]. This genotype was also associated with lung cancer and nicotine dependence in several other studies [2-5]. So far, the scientific evidence on COPD and lung function for the CHRNA3 polymorphism mostly stems from case-control studies with high smoking exposure. However, we present results from a large general-population sample. Although, in the Copenhagen City Heart Study, we previously found that the CHRNA3 rs1051730 genotype was associated with COPD hospitalisation [5] and a recent meta-analysis implicated several other polymorphisms in other genes in affecting lung function [6], the influence of this genotype on slight changes in lung function in smokers in the general population is largely unknown. Likewise, the association of this genotype with COPD of different severities and defined using different spirometric criteria is unexplored in the general population.

We first tested the hypotheses that the CHRNA3 rs1051730 genotype is associated with reduced lung function in smokers in the general population; for comparison, we also studied nonsmokers whereas previous studies were mainly in smokers $[1,7,8]$. Secondly, we tested whether the genotype was associated with COPD defined using the Global Initiative for Chronic Obstructive Lung Disease (GOLD) criteria of increasing severity (GOLD stages

\section{AFFILIATIONS}

Dept of Clinical Biochemistry and The Copenhagen General Population Study, Herlev Hospital, and Copenhagen University Hospital, Faculty of Health Sciences, University of Copenhagen, Copenhagen, Denmark.

CORRESPONDENCE

S.E. Bojesen

Dept of Clinical Biochemistry, 54M1, Herlev Hospital

Copenhagen University Hospital Herlev Ringvej 75

DK-2730 Herlev

Denmark

E-mail: Stig.Egil.Bojesen@

regionh.dk

Received:

Oct 112011

Accepted after revision:

Feb 272012

First published online:

March 222012 
I-IV, II-IV and III-IV) [9], defined by the lower limit of normal for the forced expiratory volume in $1 \mathrm{~s}$ (FEV1)/forced vital capacity (FVC) ratio [10] and defined as hospitalisation with COPD [11, 12]. To answer these questions with maximum power, we studied 57,657 individuals from the Danish general population, the Copenhagen General Population Study cohort, of whom 54,289 had spirometry performed and 34,592 were ever-smokers; this is a different sample of the Danish general population from that in our previous study [5]. In addition, the large size of our study allowed us to investigate associations with COPD severity stages. A test for an association between the rs1051730 genotype and lung cancer was included as a positive control. Finally, we tested the association between rs1051730 genotype and detailed smoking behaviour, including use of nicotinic replacement therapy in former smokers. All hypotheses were pre-specified.

\section{METHODS}

\section{Ethical aspects}

The Copenhagen General Population Study was approved by Herlev Hospital (Copenhagen, Denmark) and the scientific ethical committee for Copenhagen and Frederiksberg (H-KF01-144/01), and was conducted according to the Declaration of Helsinki. All participants gave written informed consent. The study recruited participants from a different part of Copenhagen to the Copenhagen City Heart Study used in our previous study [5].

\section{Settings and participants}

The Copenhagen General Population Study is a single-centre study of white subjects of Danish descent from the Danish general population [12-14]. The study was initiated in 2003 and is still recruiting participants aged $\geqslant 20$ yrs who are randomly selected from the national Danish Civil Registration System. All participants filled in a questionnaire, underwent a physical examination and had a blood sample drawn for DNA isolation. We included the first 57,811 participants. Of these, 83 participants were excluded due to being of an ethnicity other than Danish and a further 71 participants had missing genotype information. This left a total of 57,657 participants for analysis. The Copenhagen General Population Study is similar to the Copenhagen City Heart Study, an earlier study used our previous analyses [5], but the participants in the two studies are from different parts of Copenhagen. As the Copenhagen General Population Study was conducted at a later point in time, there were fewer smokers in the present study [15]. The response rate was $46 \%$ for the Copenhagen General Population Study.

\section{Spirometry, COPD and lung cancer diagnoses}

FEV1 and FVC (without bronchodilatation) was measured with a dry-wedge spirometer (Vitalograph, Maids Moreton, UK) in the first 15,000 participants and with an EasyOne Spirometer (Medizintechnik, Zurich, Switzerland) in the rest of the participants. No major systematic difference was observed for the two different devices regarding the distribution of lung function values. Each spirometry was performed in triplicate and results were accepted only if variation between the two best-performing of these was $<5 \%$; the best results were used.

Predicted values were calculated using multiple regression analyses separately for males and females, with age and height as covariates in never-smokers [10]. The \% pred value was calculated by dividing the observed value by the predicted value. The lower limit of normal was calculated as the difference between the predicted value and 1.645 times the standard error of the estimate, separately for males and females [16, 17]. COPD was defined in five different ways: 1 ) hospitalisation with COPD (International Classification of Diseases (ICD)-8: 491-492; ICD10: J41-J44); 2) the lower limit of normal for FEV1/FVC; 3) GOLD I-IV (FEV1/FVC <0.7); 4) GOLD II-IV (FEV1/FVC $<0.7$ and FEV1 $<80 \%$ pred); and 5) GOLD III-IV (FEV1/FVC $<0.7$ and $\mathrm{FEV} 1<50 \%$ pred). Individuals $<40$ yrs of age with self-reported asthma were omitted from analyses of COPD. Hospitalised lung cancer individuals were diagnosed with ICD-7 codes 162-164 and 462.2-462.4, and ICD-10 codes C33-C34 and C37-C38. Diagnoses on all individuals were collected from the national Danish Patient Registry from 1976 to August 8, 2010 and from the national Danish Cancer Registry from 1976 to May 17, 2009.

\section{Smoking behaviour}

The participants were divided into three groups: never-, former and current smokers. Former smokers were those who used to smoke in the past but did not at the time of the study. Eversmokers were both former and current smokers. In the questionnaire, all participants were asked about age at smoking onset and former smokers were also asked about age at smoking cessation. For former smokers, this information was used to calculate smoking duration, while similar calculations for current smokers were based on age at smoking onset and date of examination. Daily tobacco consumption was calculated in grams of tobacco per day while cumulative tobacco consumption was calculated in pack-years, defined as $20 \mathrm{~g}$ tobacco per day per year. All ever-smokers were asked about smoking inhalation, and former smokers were asked about dependence and number of years on nicotinic replacement therapy.

\section{Genotyping}

DNA from all participants were isolated from full blood and stored at $-45{ }^{\circ} \mathrm{C}$. We used the Taqman ${ }_{\circledast}$ method (Applied Biosystems Inc., Foster City, CA, USA) to genotype rs1051730 in the CHRNA3 gene. The genotype was called using SDS Taqman $₫$ allelic discrimination version 2.2.2 on the ABI PRISM 7900HT Sequence Detection System. Primers and probes are available from the authors on request. Due to re-runs, the genotyping call rate was $99.9 \%$. Control sequencing using an Applied Biosystems 3730 DNA Analyser was performed in randomly chosen samples showing 100\% agreement between the two methods. All genotyping was performed at Herlev Hospital.

\section{Statistical analyses}

Data analyses were performed using STATA/SE 11.1 (StataCorp LP, College Station, TX, USA). Analyses of lung function values were stratified according to smoking status. Tests of interaction were performed using two-way ANOVA by introducing a twofactor term. Odds ratios for COPD hospitalisation and severity outcomes were calculated using logistic regression, and adjusted for age, sex and cumulative tobacco consumption. For multifactorial adjustment, missing data for cumulative tobacco consumption $(2.8 \%)$ were imputed. To approach a normal distribution in ever-smokers, cumulative tobacco consumption was square root-transformed, FVC \% pred was transformed logarithmically, while FEV1/FVC was squared for multiple regression and ANOVA analyses; these were the transformations that most closely approached the normal distribution. 


\section{RESULTS}

A total of 57,657 participants were included in this study. Of these, $45 \%$ were noncarriers, $44 \%$ were heterozygous and $11 \%$ were homozygous for the CHRNA3 rs1051730 genotype, which is similar to values seen in previous studies [1, 2, 4]. The genotype distribution was in Hardy-Weinberg equilibrium $(p=0.25)$. Baseline characteristics did not differ by genotype (table S1). The participation by smoking status for genotyping and spirometry is shown in table S2. The distribution by smoking status of sex, age, lung function, COPD outcome and genotype is shown in table 1 .

\section{Lung function}

In homozygous, heterozygous and noncarrier ever-smokers, FEV1 was $94.1 \%$ pred, $95.3 \%$ pred and $96.5 \%$ pred, FVC was 97.1\% pred, $97.5 \%$ pred and $98.3 \%$ pred, and FEV1/FVC was $0.770,0.773$ and 0.777 , respectively (all $\mathrm{p}<0.001$ for trend; table 2). When adjusted for cumulative tobacco consumption, these associations remained significant. Also, the residuals of lung function in ever-smokers after regression with cumulative tobacco consumption showed a significant trend in the same direction (table S3). However, the interaction with cumulative tobacco consumption in ever-smokers was only significant for FEV1/FVC ( $\mathrm{p}=0.02$; table 2$)$. No differences in lung function measures across genotypes were found in never-smokers. In accordance with this, smoking status (never-/ever-smokers) and genotype interacted on FEV1 \% pred and FEV1/FVC ratio (both $\mathrm{p}<0.001$; table 2).

\section{COPD and lung cancer}

In ever-smokers, when adjusted for age and sex, genotype, from noncarriers to heterozygotes to homozygotes, was associated with increased risk of COPD, irrespective of which definition was used (all $p \leqslant 0.001$ for trend; fig. 1). The odds ratios for COPD in homozygotes versus noncarriers was 1.3 (95\% CI 1.1-1.5) for COPD hospitalisation, 1.3 (95\% CI 1.2-1.4)

\begin{tabular}{|c|c|c|}
\hline Characteristics & Ever-smokers & Never-smokers \\
\hline \multicolumn{3}{|l|}{ Sex } \\
\hline Females & $18061(52)$ & $13006(60)$ \\
\hline Males & $16531(48)$ & 8469 (39) \\
\hline Age yrs & $55(44-65)$ & $58(49-67)$ \\
\hline \multicolumn{3}{|l|}{ Lung function } \\
\hline FEV $1 \%$ pred & 95.7 (84.5-105.9) & $100.2(90.9-109.5)$ \\
\hline FVC \% pred & $97.8(87.7-107.6)$ & 99.8 (90.9-109.1) \\
\hline $\mathrm{FEV}_{1} / \mathrm{FVC}$ & 77.5 (72.3-81.8) & $80.0(75.7-83.8)$ \\
\hline \multicolumn{3}{|l|}{ COPD outcomes } \\
\hline Hospitalisation & 2077 (6) & $179(0.8)$ \\
\hline $\mathrm{FEV}_{1 / \mathrm{FVC}}<\mathrm{LLN}$ & 4227 (13) & $1074(5)$ \\
\hline GOLD I-IV & 5839 (18) & $1593(8)$ \\
\hline GOLD II-IV & 3161 (10) & 532 (3) \\
\hline GOLD III-IV & $612(2)$ & $54(0.3)$ \\
\hline \multicolumn{3}{|l|}{ Genotype } \\
\hline Noncarriers ${ }^{\#}$ & $15633(45)$ & $9490(44)$ \\
\hline Heterozygotes & $15330(44)$ & 9599 (45) \\
\hline Homozygotes $^{+}$ & 3629 (11) & 2386 (11) \\
\hline
\end{tabular}

Data are presented as $\mathrm{n}(\%)$ or median (interquartile range). FEV1: forced expiratory volume in $1 \mathrm{~s}$; \% pred: \% predicted; FVC: forced vital capacity; COPD: chronic obstructive pulmonary disease; LLN: Iower limit of normal; GOLD: Global Initiative for Chronic Obstructive Lung Disease. ${ }^{\#}$ : CC genotype; ${ }^{\circ}$ : CT genotype; ${ }^{+}$: TT genotype.

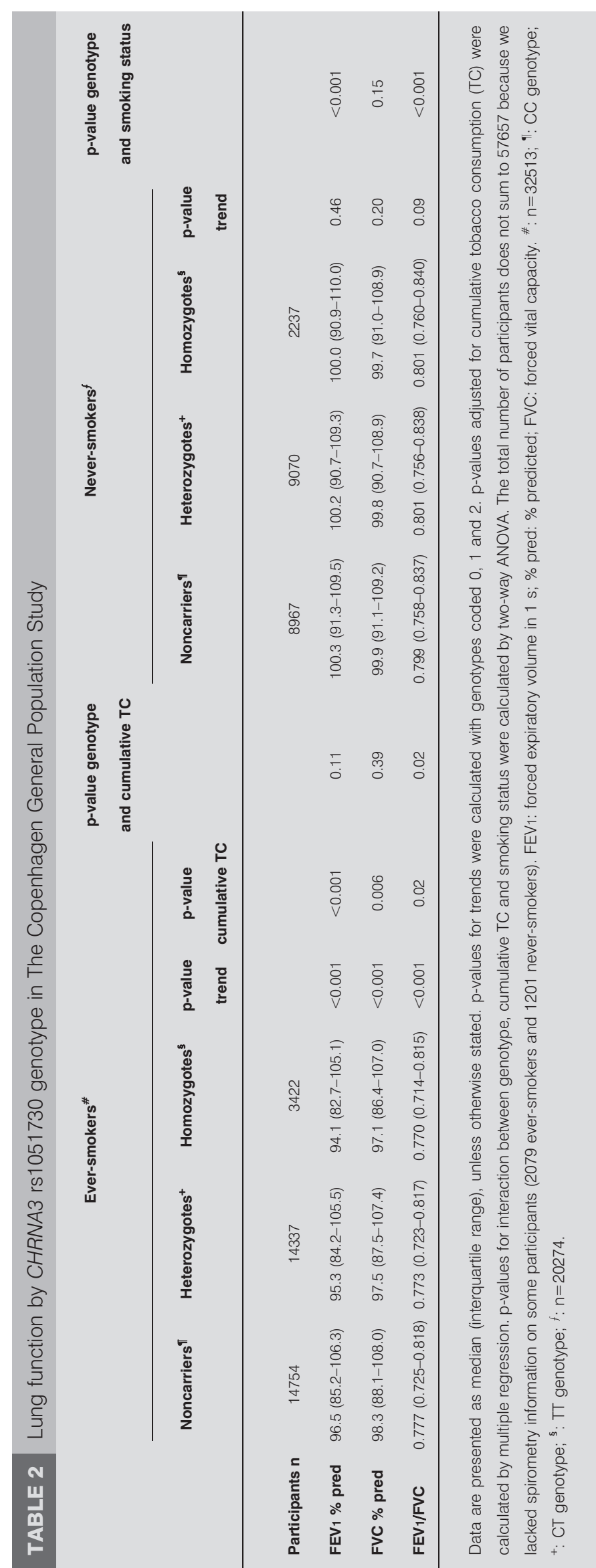




\begin{tabular}{|c|c|c|c|c|c|c|c|c|}
\hline COPD definition & Genotype & Total/events & $\begin{array}{c}\text { Age and } \\
\text { sex adjusted }\end{array}$ & $\begin{array}{c}\text { OR } \\
(95 \% \mathrm{Cl})\end{array}$ & $\begin{array}{l}p \text {-value } \\
\text { for trend }\end{array}$ & $\begin{array}{l}\text { Age, sex and } \\
\text { cumulative tobac } \\
\text { consumption } \\
\text { adjusted }\end{array}$ & $\begin{array}{c}\text { OR } \\
(95 \% \mathrm{Cl})\end{array}$ & $\begin{array}{l}p \text {-value } \\
\text { for trend }\end{array}$ \\
\hline Hospitalisation & $\begin{array}{l}\text { Noncarriers } \\
\text { Heterozygotes } \\
\text { Homozygotes }\end{array}$ & $\begin{array}{c}15548 / 880 \\
15231 / 947 \\
3609 / 247\end{array}$ & $\int_{1+1}^{\infty+1}$ & $\begin{array}{c}1.0 \\
1.1(1.0-1.2) \\
1.3(1.1-1.5)\end{array}$ & 0.001 & $i_{1-1}$ & $\begin{array}{c}1.0 \\
1.1(1.0-1.2) \\
1.1(1.0-1.3)\end{array}$ & 0.08 \\
\hline FEV $1 / F V C<L L N$ & $\begin{array}{l}\text { Noncarriers } \\
\text { Heterozygotes } \\
\text { Homozygotes }\end{array}$ & $\begin{array}{c}14677 / 1787 \\
14246 / 1904 \\
3405 / 510\end{array}$ & $\prod_{1+1}$ & $\begin{array}{c}1.0 \\
1.1(1.0-1.2) \\
1.3(1.2-1.4)\end{array}$ & $<0.001$ & $\int_{-1}^{+1}$ & $\begin{array}{c}1.0 \\
1.1(1.0-1.2) \\
1.2(1.1-1.3)\end{array}$ & 0.001 \\
\hline GOLD I-IV & $\begin{array}{l}\text { Noncarriers } \\
\text { Heterozygotes } \\
\text { Homozygotes }\end{array}$ & $\begin{array}{c}14677 / 2512 \\
14246 / 2607 \\
3405 / 699\end{array}$ & in & $\begin{array}{c}1.0 \\
1.1(1.0-1.2) \\
1.3(1.2-1.4)\end{array}$ & $<0.001$ & tor & $\begin{array}{c}1.0 \\
1.1(1.0-1.1) \\
1.2(1.1-1.3)\end{array}$ & $<0.001$ \\
\hline GOLD II-IV & $\begin{array}{l}\text { Noncarriers } \\
\text { Heterozygotes } \\
\text { Homozygotes }\end{array}$ & $\begin{array}{c}14677 / 246 \\
14246 / 272 \\
3405 / 402\end{array}$ & ind & $\begin{array}{c}1.0 \\
1.1(1.0-1.2) \\
1.4(1.2-1.6)\end{array}$ & $<0.001$ & $\sum_{101}$ & $\begin{array}{c}1.0 \\
1.1(1.0-1.2) \\
1.3(1.1-1.4)\end{array}$ & 0.001 \\
\hline GOLD III-IV & $\begin{array}{l}\text { Noncarriers } \\
\text { Heterozygotes } \\
\text { Homozygotes }\end{array}$ & $\begin{array}{c}14677 / 246 \\
14246 / 272 \\
3405 / 92\end{array}$ & $\int_{-1}$ & $\begin{array}{c}1.0 \\
1.2(1.0-1.4) \\
1.7(1.3-2.1)\end{array}$ & $<0.001$ & $\int_{1-1}$ & $\begin{array}{c}1.0 \\
1.1(0.9-1.3) \\
1.5(1.2-2.0)\end{array}$ & 0.003 \\
\hline Lung cancer & $\begin{array}{l}\text { Noncarriers } \\
\text { Heterozygotes } \\
\text { Homozygotes }\end{array}$ & $\begin{array}{c}15626 / 85 \\
15327 / 115 \\
3628 / 34\end{array}$ & $\longmapsto$ & $\begin{array}{c}1.0 \\
1.4(1.1-1.9) \\
1.8(1.2-2.6)\end{array}$ & 0.002 & $\mapsto$ & $\begin{array}{c}1.0 \\
1.3(1.0-1.8) \\
1.6(1.1-2.4)\end{array}$ & 0.009 \\
\hline \multicolumn{9}{|c|}{0.51 .0} \\
\hline
\end{tabular}

FIGURE 1. Risk of chronic obstructive pulmonary disease (COPD) by CHRNA3 genotype adjusted for age, sex and cumulative tobacco consumption in ever-smokers in the Copenhagen General Population Study. Circles represent the point estimate of the odds ratio and whiskers represent the $95 \%$ confidence interval of the estimate. p-values were calculated with the genotypes coded as 0,1 , and 2 . The total number does not sum to 34592 because individuals aged $<40$ yrs with self-reported asthma ( $n=204$ ) were excluded from analyses of COPD and because of missing spirometry information on some participants $(n=2079)$. Global Initiative for Chronic Obstructive Lung Disease (GOLD) stage I-IV was defined as forced expiratory volume in $1 \mathrm{~s}$ (FEV1)/forced vital capacity (FVC) $<0.7$. GOLD II-IV was defined as FEV1/FVC $<0.7$ and FEV $1<80 \%$ predicted. GOLD III-IV was defined as FEV1/FVC $<0.7$ and FEV1 $<50 \%$ pred. Corresponding data for never-smokers are shown in figure S1. LLN: lower limit of normal.

for COPD defined as FEV1/FVC less than the lower limit of normal, 1.3 (95\% CI 1.2-1.4) for GOLD I-IV, 1.4 (95\% CI 1.2 1.6) for GOLD II-IV and 1.7 (95\% CI 1.3-2.1) for GOLD III-IV. When further adjusted for cumulative tobacco consumption the association remained significant for all COPD definitions, except hospitalisation for COPD. Number of participants with COPD according to 10 -yr age groups and the five different COPD definitions are shown in table S4.

The odds ratio for lung cancer was 1.8 (95\% CI 1.2-2.6) for homozygotes versus noncarriers in ever-smokers when adjusted for age and sex (fig. 1). This association remained significant after adjustment for cumulative tobacco consumption.

In never-smokers, there was no association between any of the COPD definitions and genotype (fig. S1). The association between genotype and lung cancer in never-smokers was not analysed because there was only one event in the homozygous group.

\section{Smoking behaviour}

Genotype was associated with both daily and cumulative tobacco consumption in both current and former smokers (all $\mathrm{p}<0.001$ for trend; table 3). In current smokers, the daily tobacco consumption was $17.2 \mathrm{~g} \cdot \mathrm{day}^{-1}$ in homozygotes versus $15.1 \mathrm{~g} \cdot$ day $^{-1}$ in noncarriers, while the cumulative tobacco consumption was 32.1 pack-yrs in homozygotes versus 28.4 pack-yrs noncarriers. Corresponding results in former smokers for daily tobacco consumption was $15.7 \mathrm{~g} \cdot$ day $^{-1}$ in homozygotes versus $13.8 \mathrm{~g} \cdot$ day $^{-1}$ in noncarriers, while the cumulative tobacco consumption was 20.3 pack-yrs in homozygotes versus 17.4 pack-yrs in noncarriers. We found no association between genotype and age at smoking onset, smoking cessation, smoking duration or smoking inhalation when corrected for multiple comparisons using the Bonferroni method (table 3).

Genotype was associated with use of nicotinic replacement therapy in former smokers: frequency of nicotinic replacement therapy across genotypes was 5.0\% for homozygotes, $4.6 \%$ for heterozygotes and $3.5 \%$ for noncarriers $(p<0.001$ for trend; fig. 2). However, no significant association was found between genotype and years of dependence on nicotinic replacement therapy after smoking cessation, but there was a trend $(p=0.09)$.

\section{DISCUSSION}

\section{Principal findings}

First, examining 57,657 individuals in the general population, we demonstrated a reduced lung function in ever-smokers for 


\begin{tabular}{|c|c|c|c|c|c|c|}
\hline \multicolumn{2}{|c|}{ Baseline characteristics } & Participants & Noncarriers ${ }^{\#}$ & Heterozygotes" & Homozygotes $^{+}$ & $p$-value for trend \\
\hline \multicolumn{7}{|c|}{ Current smokers } \\
\hline Total & & 12089 & 5234 & 5478 & 1377 & \\
\hline Tobacco o & nsumption $\mathrm{g} \cdot$ day $^{-1}$ & 11958 & $15.1 \pm 0.1$ & $16.1 \pm 0.1$ & $17.2 \pm 0.3$ & $<0.001$ \\
\hline Age at sm & king onset yrs & 11967 & $17.9 \pm 0.1$ & $17.7 \pm 0.1$ & $17.7 \pm 0.2$ & 0.02 \\
\hline Smoking $c$ & ration yrs & 12037 & $36.4 \pm 0.2$ & $36.4 \pm 0.2$ & $36.7 \pm 0.4$ & 0.69 \\
\hline Smoking ir & lalation ${ }^{\S}$ (\%) & 10490 & $4533(87)$ & $4752(87)$ & 1205 (88) & 0.32 \\
\hline \multicolumn{7}{|c|}{ Former smokers } \\
\hline Total & & 22053 & 10200 & 9650 & 2203 & \\
\hline Tobacco o & nsumption $\mathrm{g} \cdot$ day $^{-1}$ & 21379 & $13.8 \pm 0.1$ & $14.9 \pm 0.1$ & $15.7 \pm 0.2$ & $<0.001$ \\
\hline Smoking in & nalation ${ }^{\S} \mathrm{n}(\%)$ & 17870 & 8241 (82) & 7852 (82) & 1777 (82) & 0.40 \\
\hline
\end{tabular}

CHRNA3 rs1051730 heterozygotes and homozygotes versus noncarriers. Secondly, we showed an association between genotype and COPD, regardless of whether the definition of COPD was hospitalisation, or spirometric using a fixed value for FEV1/FVC ratio and FEV1 \% pred or lower limit of normal for FEV1/FVC ratio, with the highest odds ratio for the most severe COPD, GOLD III-IV. Thirdly, we confirmed an association with lung cancer, which has been reported previously [2-5] and therefore included as a positive control of this study. Finally, we found an association with increased tobacco consumption in current and former smokers, and for the first time with nicotinic replacement therapy in former smokers.

\section{Strengths of the study}

Strengths of the present study include the following: 1) our study was well suited to address genetic effects in COPD, a complex disease, where genetic effects are expected to be rather small; 2) we had the opportunity to assess the effects on different severity grades of COPD; 3 ) we studied almost 60,000 individuals from the general population all recruited at a single centre; 4) the COPD diagnoses were not based on self-reported data, but instead on high-quality spirometric measurements and information on hospitalisation from national registries, eliminating risk of recall bias; and 5) we studied white participants only, eliminating the possibility of bias in results from population admixture of people of different ethnicities (however, we cannot completely exclude occult stratification within people of Danish descent). Nevertheless, we believe that our findings are relevant for white populations exposed to tobacco smoke.

\section{Limitations of the study}

Other polymorphisms in the region $15 \mathrm{q} 25$ have been associated with smoking behaviour and lung disease, but these were not examined in this study since they have shown linkage disequilibrium with rs1051730 [3]. However, rs1051730 is a silent polymorphism and the results observed are most likely due to linkage disequilibrium with a functional polymorphism or haplotype that probably affects the nicotinic acetylcholine receptor.

The American Thoracic Society and European Respiratory Society recommend that COPD is defined spirometrically as FEV1/FVC ratio below lower limit of normal as this definition is capable of identifying more individuals with an obstructive pattern compared with the FEV1/FVC ratio [10]. We only had the opportunity of using FEV1/FVC ratio in our analyses and, therefore, we cannot exclude that this could have affected our results slightly. Vital capacity might be higher in individuals with COPD due to collapse of narrow airways during a forced manoeuvre and the use of $\mathrm{FEV}_{1} / \mathrm{FVC}$ ratio will thus diagnose a higher number of individuals with COPD [18]. Also, as we studied white participants only, our results may not necessarily apply to other ethnicities.

We did not have the opportunity to measure lung function values after bronchodilatation due to cost limitations. This could possibly give a risk of misclassification of asthma as COPD, but as we excluded all individuals below the age of 40 yrs with asthma in order to avoid major misclassification of COPD, we do not expect that using lung function values without bronchodilatation have affected the observed association between genotype and COPD to a major extent.

Participants who were prevented from attending the study due to severe COPD or early death can distort our results due to selection bias, if the association between genotype and COPD differs for the group of individuals who participated in the study compared with those who did not participate. However, such a selection bias would probably be independent of 



FIGURE 2. Use of nicotinic replacement therapy by CHRNA3 genotype in former smokers in a) frequency ( $p<0.001$ for trend) and b) years of use ( $p=0.09$ for trend) in the Copenhagen General Population Study. $p$-values for trends were calculated with genotypes coded as 0,1 and 2 .

genotype and, therefore, would only tend to underestimate the results, and thus cannot explain the observed association. Given the very large size of the study sample, it is not very likely that a selection bias falsely produced the observed associations, as the underlying selection would have to be very strong, and probably would not go undetected while running the study.

As different methods were used to measure lung function in the first 15,000 participants compared with the rest of the participants, a possible bias could exist if the two methods were not comparable. However, as we observed no major systematic difference between the methods, we do not believe that this has distorted our results.

\section{Results in relation to other studies}

Our findings are supported by other studies reporting an association between genotype and reduced FEV1 or FEV1/FVC ratio, COPD and emphysema [5, 7, 8, 19]. However, one study of heavy smokers failed to find an association with COPD severity according to GOLD stage, but the study only reported genotype distributions in the different GOLD stages [7]. A strength of our study is the number and type of participants and that we also report risk estimates.

A recent GWAS meta-analysis found some evidence of an association between rs1051730 and lung function although a significant gene-by-smoking interaction was not reported [6]. The Copenhagen General Population Study is a single-centre study that includes a larger number of individuals by itself than the entire GWAS meta-analysis and, in our study, detailed uniform smoking information was available on all participants. It is therefore plausible that we can detect an association that previous studies did not have the power to report.

\section{Possible explanations}

Mechanistically, our findings are plausible, as the neuronal nicotinic acetylcholine receptor is expressed throughout the central nervous system and responds to release of acetylcholine but also responds to nicotine [20]. Thus, response of these receptors to nicotine in the blood from tobacco-smoke is part of the perceived positive effects of smoking [21].

The fact that the association between genotype and lung function and COPD was only present in ever-smokers raises the question of whether the apparent effect of genotype is rather due to an association through smoking behaviour. We showed that homozygous ever-smokers have a higher tobacco consumption than noncarriers. Thus, the higher tobacco consumption in this group would naturally increase their risk for lower spirometric measurements as well as a higher risk of COPD and lung cancer, which could explain our results. However, when adjusted for cumulative tobacco consumption, the associations with both spirometric measurements and diseases remained. Like in earlier studies on lung cancer, we found no association of genotype in never-smokers with lung function or COPD [2, 22-24]. This could indicate that smoking in carriers of variant alleles is necessary for developing lung function decline and disease, but that the genotype plays an additional role beyond that of the effect on smoking behaviour [22, 25]. At this time, a clear biological explanation for the direct effect still remains to be established $[1-4,19,25]$. Another explanation for the remaining associations of genotype with risk of lung disease after adjusting for smoking behaviour might be that ever-smokers under-report their smoking behaviour, and that under-reporting is more pronounced in heavy smokers.

A novel finding in the present study is that the proportion of former smokers dependent on nicotinic replacement therapy increased from noncarriers to heterozygotes to homozygotes. Our demonstration of an association between genotype and nicotine dependence is in accordance with earlier findings $[4,26,27]$. Thus, our findings further confirm that carriers of CHRNA3 variant allele indeed are more dependent on nicotine compared with noncarriers, rather than smoking per se.

\section{Conclusion and future research}

We have investigated the effects of the CHRNA3 polymorphism in a very large sample, and we could replicate associations in a general population sample that have previously been 
found in case-control studies, mostly of smokers. Also, we did not observe any associations in our large sample of $>20,000$ never-smokers, which suggests that the effects of the polymorphism are indeed likely to be present only in smokers. Finally, in ever-smokers, we found that the polymorphism is associated with important clinical outcomes such as COPD hospitalisation and severity, but also with tobacco consumption and use of nicotinic replacement therapy in former smokers. Aside from hospitalisation, these findings are new. The effects of a single polymorphism will probably have low predictive power for nicotine addiction on an individual level, but if further variants are identified in the future, this might become relevant for smoking cessation programmes. The findings in our study could indicate a possible link between smoking/nicotine dependence and important clinical outcomes that are mediated by the CHRNA3 polymorphism.

\section{SUPPORT STATEMENT}

This study was funded by the Danish Heart Foundation, the Novo Nordisk Foundation and the Danish Medical Research Council. The funding organisations had no role in design of the study, data collection, analyses or interpretation of data, the writing process or approval of the submitted manuscript. All researchers were independent from the funders.

\section{STATEMENT OF INTEREST}

None declared.

\section{ACKNOWLEDGEMENTS}

The authors thank laboratory technician K. Skjoldager (Dept of Clinical Biochemistry, Herlev Hospital, Copenhagen, Denmark). We would also like to thank the participants in The Copenhagen General Population Study for their willingness to participate.

\section{REFERENCES}

1 Pillai SG, Ge D, Zhu G, et al. A genome-wide association study in chronic obstructive pulmonary disease (COPD): identification of two major susceptibility loci. PLoS Genet 2009; 5: e1000421.

2 Amos CI, Wu X, Broderick P, et al. Genome-wide association scan of tag SNPs identifies a susceptibility locus for lung cancer at 15q25.1. Nat Genet 2008; 40: 616-622.

3 Hung RJ, McKay JD, Gaborieau V, et al. A susceptibility locus for lung cancer maps to nicotinic acetylcholine receptor subunit genes on 15q25. Nature 2008; 452: 633-637.

4 Thorgeirsson TE, Geller F, Sulem P, et al. A variant associated with nicotine dependence, lung cancer and peripheral arterial disease. Nature 2008; 452: 638-642.

5 Kaur-Knudsen D, Bojesen SE, Tybjaerg-Hansen A, et al. Nicotinic acetylcholine receptor polymorphism, smoking behavior, and tobacco-related cancer and lung and cardiovascular diseases: a cohort study. J Clin Oncol 2011; 29: 2875-2882.

6 Hancock DB, Eijgelsheim M, Wilk JB, et al. Meta-analyses of genome-wide association studies identify multiple loci associated with pulmonary function. Nat Genet 2010; 42: 45-52.

7 Lambrechts D, Buysschaert I, Zanen P, et al. The 15q24/25 susceptibility variant for lung cancer and chronic obstructive pulmonary disease is associated with emphysema. Am J Respir Crit Care Med 2010; 181: 486-493.

8 Saccone NL, Culverhouse RC, Schwantes-An TH, et al. Multiple independent loci at chromosome 15q25.1 affect smoking quantity: a meta-analysis and comparison with lung cancer and COPD. PLoS Genet 2010; 6: e1001053.
9 Rabe KF, Hurd S, Anzueto A, et al. Global strategy for the diagnosis, management, and prevention of chronic obstructive pulmonary disease: GOLD executive summary. Am J Respir Crit Care Med 2007; 176: 532-555.

10 Pellegrino R, Viegi G, Brusasco V, et al. Interpretative strategies for lung function tests. Eur Respir J 2005; 26: 948-968.

11 Baekvad-Hansen M, Dahl M, Tybjaerg-Hansen A, et al. Surfactant protein-B 121ins2 heterozygosity, reduced pulmonary function, and chronic obstructive pulmonary disease in smokers. Am J Respir Crit Care Med 2010; 181: 17-20.

12 Dahl M, Bowler RP, Juul K, et al. Superoxide dismutase 3 polymorphism associated with reduced lung function in two large populations. Am J Respir Crit Care Med 2008; 178: 906-912.

13 Kamstrup PR, Tybjaerg-Hansen A, Steffensen R, et al. Genetically elevated lipoprotein(a) and increased risk of myocardial infarction. JAMA 2009; 301: 2331-2339.

14 Zacho J, Tybjaerg-Hansen A, Jensen JS, et al. Genetically elevated $\mathrm{C}$-reactive protein and ischemic vascular disease. $N$ Engl J Med 2008; 359: 1897-1908.

15 Kaur-Knudsen D, Nordestgaard BG, Tybjaerg-Hansen A, et al. CYP1B1 genotype and risk of cardiovascular disease, pulmonary disease, and cancer in 50,000 individuals. Pharmacogenet Genomics 2009; 19: 685-694.

16 Aggarwal AN, Gupta D, Behera D, et al. Comparison of fixed percentage method and lower confidence limits for defining limits of normality for interpretation of spirometry. Respir Care 2006; 51: $737-743$

17 Swanney MP, Ruppel G, Enright PL, et al. Using the lower limit of normal for the FEV1/FVC ratio reduces the misclassification of airway obstruction. Thorax 2008; 63: 1046-1051.

18 Chhabra SK. Forced vital capacity, slow vital capacity, or inspiratory vital capacity: which is the best measure of vital capacity? J Asthma 1998; 35: 361-365.

19 Pillai SG, Kong X, Edwards LD, et al. Loci identified by genomewide association studies influence different disease-related phenotypes in chronic obstructive pulmonary disease. Am J Respir Crit Care Med 2010; 182: 1498-1505.

20 Saccone SF, Hinrichs AL, Saccone NL, et al. Cholinergic nicotinic receptor genes implicated in a nicotine dependence association study targeting 348 candidate genes with 3713 SNPs. Hum Mol Genet 2007; 16: 36-49.

21 Benowitz NL. Neurobiology of nicotine addiction: implications for smoking cessation treatment. Am J Med 2008; 121: S3-S10.

22 Spitz MR, Amos CI, Dong Q, et al. The CHRNA5-A3 region on chromosome $15 \mathrm{q} 24-25.1$ is a risk factor both for nicotine dependence and for lung cancer. J Natl Cancer Inst 2008; 100: $1552-1556$.

23 Galvan A, Dragani TA. Nicotine dependence may link the $15 \mathrm{q} 25$ locus to lung cancer risk. Carcinogenesis 2010; 31: 331-333.

24 Truong T, Hung RJ, Amos CI, et al. Replication of lung cancer susceptibility loci at chromosomes 15q25, 5p15, and 6p21: a pooled analysis from the International Lung Cancer Consortium. J Natl Cancer Inst 2010; 102: 959-971.

25 Wang J, Spitz MR, Amos CI, et al. Mediating effects of smoking and chronic obstructive pulmonary disease on the relation between the CHRNA5-A3 genetic locus and lung cancer risk. Cancer 2010; 116: 3458-3462.

26 The Tobacco and Genetics Consortium. Genome-wide metaanalyses identify multiple loci associated with smoking behavior. Nat Genet 2010; 42: 441-447.

27 Liu JZ, Tozzi F, Waterworth DM, et al. Meta-analysis and imputation refines the association of $15 \mathrm{q} 25$ with smoking quantity. Nat Genet 2010; 42: 436-440. 